\title{
Clinical Benefit-Risk Profile of Lenalidomide in Patients With Lower-risk Myelodysplastic Syndromes Without del(5q): Results of a Phase III Trial
}

\author{
Guillermo Garcia-Manero, ${ }^{1}$ Antonio Almeida, ${ }^{2}$ Pierre Fenaux, ${ }^{3}$ \\ Norbert Gattermann, ${ }^{4}$ Aristoteles Giagounidis, ${ }^{5}$ Stuart L. Goldberg, ${ }^{6}$ Keiya Ozawa, ${ }^{7}$ \\ Jerry Weaver, ${ }^{8}$ Valeria Santini ${ }^{9}$
}

Potential benefits of any therapy must be weighed against potential risks. Among patients with non-del(5q) lower-risk myelodysplastic syndromes, the rate of clinical benefit was significantly higher with lenalidomide versus placebo. Despite the occurrence of hematologic adverse events, the benefit-risk profile favored lenalidomide over placebo. Managing hematologic adverse events by dose reductions can help patients remain on treatment and achieve clinical benefit.

Background: In the phase III MDS-005 study of patients with lower-risk, non-del(5q) myelodysplastic syndromes, lenalidomide was associated with a higher rate of $\geq 8$ weeks red blood cell transfusion independence (RBC-TI) compared with placebo, but also with a higher risk of hematologic adverse events (AEs). Patients and Methods: This analysis evaluated the ratio of clinical benefit-risk in patients treated with lenalidomide or placebo, and assessed the effect of lenalidomide dose reductions on response. Clinical benefit was a composite endpoint defined as RBC-TI, transfusion reduction $\geq 4$ units packed red blood cells, hemoglobin increase $\geq 1.5 \mathrm{~g} / \mathrm{dL}$, or cytogenetic response. Results: The rate of clinical benefit was higher with lenalidomide than with placebo (31.9\% vs. $3.8 \%)$. The ratio of response (RBC-TI and clinical benefit) to risk (hematologic AEs) favored lenalidomide over placebo. Patients who underwent $\geq 1$ lenalidomide dose reduction had a longer duration of treatment, received a higher cumulative dose, and were more likely to experience clinical benefit versus patients without dose reductions. Conclusion: Despite the occurrence of hematologic AEs, the overall benefit-risk profile supported lenalidomide treatment. Appropriate management of hematologic AEs by dose reductions may help patients with myelodysplastic syndromes to remain on treatment and achieve clinical benefit.

Clinical Lymphoma, Myeloma \& Leukemia, Vol. 19, No. 4, 213-9 ๔ 2018 Published by Elsevier Inc. Keywords: Adverse events, Benefit-risk ratio, Dose reduction, Response, Treatment exposure

\section{Introduction}

Supportive care remains the standard treatment approach for anemia associated with lower-risk myelodysplastic syndromes (MDS). ${ }^{1}$ Erythropoiesis-stimulating agents (ESAs) are often first-line therapy for patients with non-del(5q) lower-risk MDS. Although

${ }^{1}$ Department of Leukemia, MD Anderson Cancer Center, Houston, TX

${ }^{2}$ Department of Hematology, Hospital da Luz, Lisbon, Portugal

${ }^{3}$ Service d'Hématologie Séniors, Hôpital Saint-Louis, Université Paris 7, Paris, France

${ }^{4}$ Klinik für Hämatologie, Onkologie und Klinische Immunologie, Heinrich-Heine-

Universität, Düsseldorf, Germany

${ }^{5}$ Department of Oncology, Hematology and Palliative Care, Marien Hospital

Düsseldorf, Düsseldorf, Germany

${ }^{6}$ John Theurer Cancer Center, Hackensack University Medical Center,

Hackensack, NJ
ESAs are not approved for this indication by the United States Food and Drug Administration, epoetin alfa has been licensed for the treatment of MDS in the European Union. Treatment with ESAs leads to erythroid hematologic improvement in $15 \%$ to $63 \%$ of patients, ${ }^{2-4}$ with a median response duration of 7 to 28 months. ${ }^{2,4}$

The Institute of Medical Science, The University of Tokyo, Tokyo, Japan

${ }^{8}$ Celgene Corporation, Summit, NJ

${ }^{9} \mathrm{MDS}$ Unit, AOU Careggi, University of Florence, Florence, Italy

Submitted: Oct 29, 2018; Accepted: Dec 15, 2018; Epub: Dec 21, 2018

Address for correspondence: Guillermo Garcia-Manero, MD, Department of

Leukemia, MD Anderson Cancer Center, 1515 Holcombe Blvd, Houston, TX 77030 E-mail contact: ggarciam@mdanderson.org 


\section{Clinical Benefit-Risk Profile of Lenalidomide in Lower-Risk Non-del(5q) MDS}

Response to ESAs can be predicted based on baseline erythropoietin (EPO) level and transfusion history. ${ }^{5}$ In the event of ESA failure, few treatment options are available that can provide prolonged reductions in red blood cell (RBC) transfusion requirement.

The efficacy and safety of lenalidomide was evaluated in RBC transfusion dependent (RBC-TD) patients with International Prognostic Scoring System (IPSS) Low- or Intermediate (Int)-1-risk non-del(5q) MDS who were ineligible for or refractory to ESAs in the phase III MDS-005 study. ${ }^{6}$ In this study, a statistically significant and clinically relevant proportion of lenalidomide-treated patients achieved RBC transfusion independence (RBC-TI) lasting $\geq 8$ weeks compared with placebo $(26.9 \%$ vs. $2.5 \%$; $P<.001)$. Achievement of RBC-TI is a particularly meaningful endpoint for patients with RBC-TD anemia, as prolonged transfusion dependence is associated with transfusion-related comorbidities $^{7}$ and considerably impaired health-related quality of life (HRQoL). ${ }^{8}$ In the MDS-005 study, achievement of RBC-TI $\geq 8$ weeks was associated with significant improvements in HRQoL $(P<.01)$. Other important measures of treatment response, such as reduction in transfusion burden or increase in hemoglobin $(\mathrm{Hb})$, also represent clinically meaningful improvements in anemia and anemia-related symptoms for patients with RBC-TD MDS.

When assessing clinical benefit, the most prominent adverse drug reactions should also be taken into account in order to define the most favorable balance between benefit and risk. The most common treatment-emergent adverse events (TEAEs) reported in the MDS005 study were hematologic, consistent with the known TEAE profile of lenalidomide in MDS. ${ }^{9-12}$ Adverse events (AEs) can negatively impact HRQoL and lead to drug discontinuation. Appropriate management of TEAEs, including dose reductions, is therefore essential for preventing early discontinuation and maintaining patients on therapy.

This analysis of data from the MDS-005 study aimed to compare the benefit-risk profiles of lenalidomide and placebo, and evaluate the relationship between lenalidomide exposure, including dose reductions and duration of treatment, and clinical benefit in patients with lower-risk, non-del(5q) MDS.

\section{Patients and Methods Study Design and Treatment}

Full details of patient eligibility criteria and study design have been described previously. ${ }^{6}$ Briefly, eligible patients were aged $\geq 18$ years, had transfusion-dependent anemia owing to IPSS Low- or Int-1-risk MDS, had non-del(5q) karyotype, and were ineligible for or refractory to ESAs. Patients were considered RBC-TD if they had an average transfusion requirement of $\geq 2$ units packed RBCs (pRBCs) per 28 days and no 8 consecutive weeks without RBC transfusions in the 16 weeks before randomization. Patients were considered refractory to ESAs if they were RBC-TD despite ESA treatment $(\geq 40,000$ units/week of recombinant human EPO for 8 weeks, or equivalent dose of darbepoetin). Patients were considered ineligible for ESAs if their serum EPO level was $>500 \mathrm{mU} / \mathrm{mL}$ with no prior ESA exposure.

Patients were randomized 2:1 to receive either oral lenalidomide $10 \mathrm{mg}$ or matching placebo once daily (both on 28-day cycles). The starting dose of lenalidomide was $5 \mathrm{mg}$ daily for patients with creatinine clearance $\geq 40$ to $<60 \mathrm{~mL} / \mathrm{min}$. Patients with
RBC-TI $\geq 8$ weeks or erythroid response by week 24 continued double-blind treatment until erythroid relapse, disease progression, unacceptable toxicity, or consent withdrawal. The trial was registered at ClinicalTrials.gov, trial number NCT01029262.

\section{Endpoints}

The primary endpoint of the MDS-005 study was the rate of RBC-TI for $\geq 8$ consecutive weeks. Secondary endpoints included RBC-TI $\geq 24$ weeks, duration of RBC-TI, time to onset of RBC-TI, erythroid response, HRQoL, and safety. Cytogenetic response (CyR) was assessed in patients with baseline cytogenetic abnormalities and $\geq 1$ follow-up assessment by central review using conventional metaphase cytogenetic analysis according to International Working Group 2006 criteria. $^{13}$ Major CyR was defined as absence of cytogenetic abnormalities if preexisting abnormalities were present; minor CyR was defined as $\geq 50 \%$ reduction in abnormal metaphases. AEs were coded using the Medical Dictionary for Regulatory Activities (MedDRA) version 16.1 and graded according to the National Cancer Institute Common Terminology Criteria for Adverse Events (NCI-CTCAE) version 3.0. Lenalidomide dose modifications or interruptions related to AEs were predefined in the study protocol. ${ }^{6}$ Cumulative dose, dose reductions, and discontinuations were recorded as part of the original study.

For this analysis, the composite endpoint of clinical benefit consisted of any of the following responses: RBC-TI $\geq 8$ consecutive weeks; transfusion reduction of $\geq 4$ units pRBCs $\geq 8$ weeks $^{13}$; $\mathrm{Hb}$ increase of $\geq 1.5 \mathrm{~g} / \mathrm{dL} \geq 8$ weeks $^{13}$; or CyR. ${ }^{13}$ The rate of transfusion reduction $\geq 4$ units pRBCs for $\geq 8$ weeks was calculated based on data collected during a 16 -week period. The timing of assessments for clinical benefit response is shown in Supplemental Figure 1 (in the online version). Evaluation of CyR was also conducted independently of the composite endpoint. The data cutoff date for this analysis was March 17, 2014.

\section{Statistical Analysis}

For each treatment group, the proportion of patients achieving a response (RBC-TI $\geq 8$ weeks or clinical benefit) was assessed. Clinical benefit was defined as the proportion of patients who met $\geq 1$ of the clinical benefit composite endpoint criteria. Patients who met multiple criteria were only counted once. The statistical assessment of clinical benefit assumed a binomial distribution for the composite endpoint. For both RBC-TI $\geq 8$ weeks and clinical benefit, 95\% confidence intervals (CIs) were calculated using a generalized linear model for binomial distributed data. Risk differences were estimated using an identity link function, relative risks were estimated using a log link function, and odds ratios (ORs) were estimated using a logit link function.

An evaluation of cumulative lenalidomide exposure, duration of treatment, and incidence and duration of response (RBC-TI $\geq 8$ weeks and clinical benefit) was performed in relation to the incidence of lenalidomide dose reductions.

At the individual patient level, cumulative mean benefit-risk ratios were calculated for each cycle using a bivariate generalized linear model assuming a binomial distribution with a log link function. Benefit was defined in 2 ways: the cumulative proportion of patients meeting the criteria for the primary endpoint of RBC-TI $\geq 8$ weeks, and the cumulative proportion of patients meeting the criteria for the 
Table 1 Baseline Characteristics of Patients in the MDS-005 Study

\begin{tabular}{|c|c|c|c|}
\hline Characteristic & Lenalidomide $(n=160)$ & Placebo $(n=79)$ & Overall $(\mathrm{N}=239)$ \\
\hline Median age, y (range) & $71.0(46.0-87.0)$ & $70.0(43.0-87.0)$ & $71.0(43.0-87.0)$ \\
\hline Male, n (\%) & $108(67.5)$ & $54(68.4)$ & $162(67.8)$ \\
\hline Median time since diagnosis, y (range) & $2.7(0.1-29.6)$ & $2.6(0.3-20.2)$ & $2.6(0.1-29.6)$ \\
\hline Median pRBC transfusion burden, units/28 d (range) & $3.0(1.8-8.8)$ & $3.3(1.5-9.8)$ & $3.0(1.5-9.8)$ \\
\hline \multicolumn{4}{|l|}{ IPSS risk category, $\mathrm{n}(\%)$} \\
\hline Low & $85(53.1)$ & $30(38.0)$ & $115(48.1)$ \\
\hline Intermediate-1 & $75(46.9)$ & $49(62.0)$ & $124(51.9)$ \\
\hline Prior MDS therapy, n (\%) & $135(84.4)$ & $65(82.3)$ & $200(83.7)$ \\
\hline Prior ESA treatment, $\mathrm{n}(\%)$ & $125(78.1)$ & $63(79.7)$ & $188(78.7)$ \\
\hline Prior G-CSF use, n (\%) & $27(16.9)$ & $11(13.9)$ & $38(15.9)$ \\
\hline \multicolumn{4}{|l|}{ Serum EPO level at screening, $n(\%)$} \\
\hline$\leq 500 \mathrm{mU} / \mathrm{mL}$ & $97(60.6)$ & $50(63.3)$ & $147(61.5)$ \\
\hline$>500 \mathrm{mU} / \mathrm{mL}$ & $58(36.3)$ & $28(35.4)$ & $86(36.0)$ \\
\hline Missing & $5(3.1)$ & $1(1.3)$ & $6(2.5)$ \\
\hline \multicolumn{4}{|l|}{ Ring sideroblast status, ${ }^{a}$ n (\%) } \\
\hline$<15 \%$ & $52(32.5)$ & $19(24.1)$ & $71(29.7)$ \\
\hline$\geq 15 \%$ & $108(67.5)$ & $60(75.9)$ & $168(70.3)$ \\
\hline Median bone marrow blast, \% (range) & $3.0(0.0-9.5)$ & $2.5(0.0-8.0)$ & $2.5(0.0-9.5)$ \\
\hline Median ANC, $\times 10^{9} / \mathrm{L}$ (range) & $2.4(0.5-12.2)$ & $2.1(0.3-10.6)$ & $2.3(0.3-12.2)$ \\
\hline Median platelet count, $\times 10^{9} / \mathrm{L}$ (range) & $243.5(43.0-746.0)$ & $233.0(60.0-1063.0)$ & $239.0(43.0-1063.0)$ \\
\hline Median hemoglobin, g/dL (range) & $8.7(5.5-12.3)$ & $8.6(5.6-11.8)$ & $8.7(5.5-12.3)$ \\
\hline
\end{tabular}

Abbreviations: ANC $=$ absolute neutrophil count; $\mathrm{EPO}=$ erythropoietin; $\mathrm{ESA}=$ erythropoiesis-stimulating agent; G-CSF $=$ granulocyte colony-stimulating factor; IPSS $=$ International Prognostic Scoring System; MDS = myelodysplastic syndromes; $\mathrm{pRBC}=$ packed red blood cells; RARS = refractory anemia with ring sideroblasts; RCMD = refractory cytopenia and multilineage dysplasia. ${ }^{a}$ For patients with RARS or RCMD.

composite endpoint of clinical benefit. Risk was defined as the cumulative proportion of patients with $\geq 1$ hematologic TEAE. In the MDS-005 study, grade 3 to 4 nonhematologic TEAEs were rare, 6,12 and nonhematologic TEAEs were therefore not included in the benefit-risk analysis. The probability of assessing a favorable benefitrisk ratio of the lenalidomide group relative to the placebo group was calculated by fitting the bivariate generalized linear model to the data via generalized estimating equations at each cycle, ${ }^{14,15}$ as described in greater detail in the Supplemental Methods (in the online version).

Adjusted mean platelet levels during cycles 1 to 4 were assessed according to response (RBC-TI $\geq 8$ weeks) using a linear mixed model for repeated measures, as described in the Supplemental Methods (in the online version).

\section{Results}

\section{Baseline Patient Characteristics}

From February 2010 to June 2013, 239 patients were enrolled at 72 treatment centers and randomized to lenalidomide $(\mathrm{n}=160)$ or placebo $(\mathrm{n}=79)$. Baseline characteristics were well-balanced across treatment groups (Table 1). At baseline, patients were receiving a median of 3.0 units of pRBC per 28 days (range, 1.5-9.8 units), $78.7 \%$ of patients had received prior ESAs, and $36.0 \%$ had a serum EPO level $>500 \mathrm{mU} / \mathrm{mL}$.

\section{Clinical Benefit $\geq 8$ Weeks}

Lenalidomide was associated with a higher proportion of patients achieving the composite endpoint of clinical benefit, compared with placebo $(31.9 \%$ vs. $3.8 \%$ ) (Table 2) as well as higher proportions meeting each criterion of the composite endpoint (achievement of RBC-TI $\geq 8$ weeks, reduction in transfusion burden of $\geq 4$ pRBC units $\geq 8$ weeks, Hb increase of $\geq 1.5 \mathrm{~g} / \mathrm{dL}$ for $\geq 8$ weeks, and CyR).

\section{CyR}

Among 43 patients evaluable for CyR, 9 (33.3\%) of 27 patients in the lenalidomide group and none $(0 \%)$ of 16 patients in the placebo group achieved CyR. Of these 9 patients, 5 (55.6\%) achieved a major response and $4(44.4 \%)$ achieved a minor response as best response. Five (55.6\%) of the 9 responders also achieved RBC-TI $\geq 8$ weeks. All 5 patients who achieved a major CyR had the +8 abnormality at baseline (Table 3 ).

\section{Lenalidomide Dose Reduction and Treatment Discontinuation}

Of the 160 patients treated with lenalidomide, 77 (48.1\%) underwent $\geq 1$ dose reduction. The median time to first dose reduction was 85 days (interquartile range [IQR], 50-114). Baseline characteristics were comparable between patients with or without dose reductions (see Supplemental Table 1 in the online version). Most dose reductions were implemented in response to TEAEs: 102 dose reductions in $73(45.6 \%)$ patients were attributed to TEAEs. Of these TEAE-related dose reductions, $47(46.1 \%)$ were due to neutropenia, $33(32.4 \%)$ to thrombocytopenia, and $2(2.0 \%)$ to other hematologic conditions. Nonhematologic TEAEs leading to 


\section{Clinical Benefit-Risk Profile of Lenalidomide in Lower-Risk Non-del(5q) MDS}

\begin{tabular}{|c|c|c|c|c|}
\hline Response, n (\%) & $\begin{array}{l}\text { Lenalidomide } \\
\qquad(n=160)\end{array}$ & $\begin{array}{c}\text { Placebo } \\
(n=79)\end{array}$ & $\begin{array}{l}\text { Odds Ratio } \\
\text { (95\% Cl) }\end{array}$ & $\begin{array}{l}\text { Relative Risk } \\
\text { (95\% Cl) }\end{array}$ \\
\hline Clinical benefit & $51(31.9)$ & $3(3.8)$ & $11.85(3.57-39.38)$ & $8.39(2.70-26.06)$ \\
\hline $\mathrm{RBC}-\mathrm{TI} \geq 8$ weeks & $43(26.9)$ & $2(2.5)$ & $14.15(3.33-60.12)$ & $10.62(2.64-42.70)$ \\
\hline Transfusion reduction $\geq 4 \mathrm{pRBC}$ units $\geq 8$ weeks & $34(21.3)$ & 0 & $N / A^{b}$ & $N / A^{b}$ \\
\hline $\mathrm{Hb}$ increase $\geq 1.5 \mathrm{~g} / \mathrm{dL} \geq 8$ weeks & $31(19.4)$ & $2(2.5)$ & $9.25(2.15-39.74)$ & $7.65(1.88-31.17)$ \\
\hline CyR & $9(5.6)$ & 0 & $N / A^{b}$ & $N / A^{b}$ \\
\hline
\end{tabular}

Abbreviations: $\mathrm{Cl}=$ confidence interval; $\mathrm{CyR}=$ cytogenetic response; $\mathrm{Hb}=$ hemoglobin; $\mathrm{N} / \mathrm{A}=$ not applicable; $\mathrm{pRBC}=$ packed red blood cells; $\mathrm{RBC}-\mathrm{TI}=$ red blood cell transfusion independence. ${ }^{\text {aD }}$ ata normalized to 8 weeks using data from a 16-week assessment period divided by 2 .

${ }^{b}$ Odds ratio and relative risk cannot be reliably estimated due to 0 events in the placebo group.

dose reductions included $3(2.9 \%)$ occurrences of asthenia and 3 (2.9\%) occurrences of rash.

TEAEs led to discontinuation of lenalidomide in 51 (31.9\%) patients; 23 (14.4\%) of these patients discontinued before the end of cycle 3. The most common AEs leading to discontinuation, occurring in $\geq 1.5 \%$ of patients, were thrombocytopenia $(8.8 \%)$, neutropenia (4.4\%), and deep vein thrombosis (1.9\%).

\section{Lenalidomide Dose Adjustments and Treatment Exposure}

Of the 77 patients who had $\geq 1$ dose reduction, $6(7.8 \%)$ had a dose reduction in the first cycle and 37 (48.1\%) had dose reductions during cycles 1 to 3 (Table 4). During the first 3 cycles, patients with $\geq 1$ dose reduction received a lower median dose than those with no dose reduction $(385 \mathrm{mg}$ [IQR, 318-520 mg] vs. $660 \mathrm{mg}$ [IQR, 415-840 mg]). However, when analyzed over the course of the study, patients with $\geq 1$ dose reduction received a higher median cumulative dose than those with no dose reductions $(950 \mathrm{mg}$ [IQR, 595-1435 mg] vs. $715 \mathrm{mg}$ [IQR, 370-1660 mg]). Similarly, duration of treatment was longer for patients with $\geq 1$ dose reduction compared with those with no dose reductions (172 days [IQR, 140-391 days] vs. 92 days [IQR, 46-168 days]).

\section{Lenalidomide Exposure and Response}

The relationship between lenalidomide dose reductions and achievement of either RBC-TI $\geq 8$ weeks or clinical benefit is shown in Table 5. Patients with $\geq 1$ dose reduction were more likely to achieve RBC-TI $\geq 8$ weeks than patients without dose

\begin{tabular}{|c|c|c|}
\hline Table 3 & $\begin{array}{l}\text { Cytogenetic Abnormalities a } \\
\text { With Cytogenetic Response }\end{array}$ & eline in Patients \\
\hline Patient & $\begin{array}{l}\text { Cytogenetic } \\
\text { Response }\end{array}$ & Baseline Karyotype \\
\hline 1 & Major & $47, X X,+8$ \\
\hline 2 & Major & $47, X Y,+8$ \\
\hline 3 & Major & $47, X Y,+8$ \\
\hline 4 & Major & $47, X Y,+8^{a}$ \\
\hline 5 & Major & $47, X Y,+8$ \\
\hline 6 & Minor & $47, X X,+8$ \\
\hline 7 & Minor & $47, X X,+8$ \\
\hline 8 & Minor & 46, XX, del(12q) \\
\hline 9 & Minor & $45, X,-Y$ \\
\hline
\end{tabular}

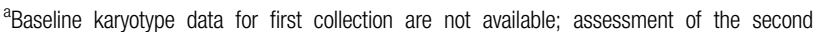
collection is reported instead. reductions (39\% vs. 16\%; OR, 3.44; 95\% CI, 1.63-7.26). Among patients who achieved RBC-TI $\geq 8$ weeks, the median duration of response was longer in patients who underwent $\geq 1$ dose reduction than in those who did not (29.6 weeks [range, 8.1-123.7 weeks] vs. 23.7 weeks [range, 9.9-103.7 weeks]). Similarly, patients with $\geq 1$ dose reduction were also more likely to achieve clinical benefit $\geq 8$ weeks (47\% vs. $18 \%$; OR, 3.98; 95\% CI, 1.94-8.15).

\section{Clinical Benefit-Risk Ratio}

The benefit-risk ratio per cycle for each treatment group is shown in Figure 1. For cycles 1 to 7, the benefit-risk ratio, in terms of the proportion of patients achieving RBC-TI $\geq 8$ weeks relative to the risk of hematologic TEAEs, favored lenalidomide treatment over placebo (Figure 1A). The probability that the RBC-TI $\geq 8$ weeksrisk ratio was relatively greater for lenalidomide than for placebo was 0.83 in cycle 1 ; this probability increased to 0.90 by cycle 4 . Similarly, the ratio of clinical benefit-risk favored the lenalidomide arm over the placebo arm (Figure 1B). In cycle 1, the probability that the clinical benefit-risk ratio was relatively greater for lenalidomide than for placebo was 0.91 , and remained fairly consistent throughout the other cycles evaluated.

\section{Relationship Between Hematologic Toxicity and Response}

A decrease in mean platelet levels (after adjusting for baseline values as a covariate) did not predict achievement of RBC-TI $\geq 8$ weeks in the MDS-005 study population (Figure 2). An exploratory analysis in patients with or without isolated anemia also showed no relationship between platelet level and achievement of RBC-TI $\geq 8$

\section{Table 4 Lenalidomide Exposure in Patients With or Without Reductions}

\begin{tabular}{l|c|c} 
Characteristic & $\geq 1$ Dose Reduction & No Dose Reduction \\
$\begin{array}{l}\text { Cumulative dose in } \\
\text { cycle 1, mg }\end{array}$ & $n=6$ & $n=154$ \\
$\quad$ Median (IQR) & $145(77.5-160.0)$ & $280(150.0-280.0)$ \\
$\begin{array}{l}\text { Cumulative dose in } \\
\text { cycles 1-3, mg } \\
\text { Median (IQR) }\end{array}$ & $n=37$ & $n=123$ \\
Total cumulative dose, mg & $385(317.5-520.0)$ & $660(415.0-840.0)$ \\
Median (IQR) & $n=77$ & $n=83$ \\
Duration of treatment, d & $950(595.0-1435.0)$ & $715(370.0-1660.0)$ \\
$\quad$ Median (IQR) & $n=77$ & $n=83$ \\
\hline
\end{tabular}

Abbreviation: $I Q R=$ interquartile range. 


\section{Guillermo Garcia-Manero et al}

Table 5 Response to Lenalidomide by Incidence of Dose Reductions

\begin{tabular}{l|c|c|c|c|c}
$\mathbf{2 1}$ Dose Reduction & $\begin{array}{c}\text { No Dose } \\
\text { Response, n/N (\%) }\end{array}$ & $\begin{array}{c}\text { Risk Difference, \% } \\
\text { (95\% Cl) }\end{array}$ & $\begin{array}{c}\text { Odds Ratio } \\
\text { (95\% CI) }\end{array}$ & $\begin{array}{c}\text { Relative Risk } \\
\text { Ratio (95\% Cl) }\end{array}$ \\
RBC-TI $\geq 8$ w & $30 / 77(39)$ & $13 / 83(16)$ & $23(10-37)$ & $3.44(1.63-7.26)$ & $2.49(1.40-4.41)$ \\
Clinical benefit & $36 / 77(47)$ & $15 / 83(18)$ & $29(15-43)$ & $3.98(1.94-8.15)$ & $2.59(1.54-4.34)$ \\
\hline
\end{tabular}

Abbreviations: $\mathrm{Cl}=$ confidence interval; $\mathrm{RBC}-\mathrm{TI}=$ red blood cell transfusion independence.

weeks (see Supplemental Figure 2 in the online version). In lenalidomide-treated patients with isolated anemia, rates of RBC-TI $\geq 8$ weeks (26.8\%) and clinical benefit (30.9\%) were consistent with the overall population $(26.9 \%$ and $31.9 \%$, respectively) (Table 2).

\section{Discussion}

In this analysis of RBC-TD patients with lower-risk non-del(5q) MDS ineligible for or refractory to ESAs, lenalidomide was associated with significantly greater clinical benefit compared with placebo. This newly defined composite endpoint of clinical benefit comprised the clinically meaningful endpoints of reduced transfusion burden, increased $\mathrm{Hb}$, and $\mathrm{CyR}$ in addition to achievement of RBC-TI. Both RBC-TI and Hb level have been shown to be important predictors of HRQoL in MDS. ${ }^{8,16,17}$ At the individual patient level, some patients may respond by one clinical definition but not by another; it is therefore important to consider these other indicators of therapeutic benefit when making treatment decisions.

We found that patients who underwent $\geq 1$ lenalidomide dose reduction were more likely to achieve RBC-TI and clinical benefit than patients with no dose reductions. This apparent disparity can be explained by the finding that patients undergoing $\geq 1$ dose reduction also had a longer duration of treatment, leading to a higher cumulative treatment exposure. Employing lenalidomide dose reductions instead of treatment discontinuation in response to AEs may allow patients to remain on treatment longer, leading to increased drug exposure and better outcomes. In MDS-005, the median time to onset of response was 10.1 weeks $^{6}$; therefore, patients who discontinued treatment within the first 3 cycles may not have received a sufficient duration of therapy to achieve a response. Previous findings in patients with $\operatorname{del}(5 \mathrm{q})$ MDS support this finding: in the MDS-003 and MDS-004 studies, lenalidomide dose reductions were associated with improved leukemia-free survival and overall survival outcomes. ${ }^{18}$

Maintaining the highest tolerated dose may also be important to patient outcomes. In a pooled analysis of data from phase II and III studies of lenalidomide in patients with $\operatorname{del}(5 \mathrm{q})$ MDS, receipt of a higher dose in cycle 1 was associated with improved short- and longterm responses. ${ }^{18,19}$ In the non-del(5q) population of the MDS-005 study, a preliminary analysis showed that more patients who received a higher cumulative lenalidomide dose in cycle $1(>210$ $\mathrm{mg})$ achieved RBC-TI $\geq 8$ weeks $(36 / 105$ [34.3\%]) and clinical benefit $(42 / 105[40.0 \%])$, compared with patients receiving $\leq 210$ mg (7/55 [12.7\%] and 9/55 [16.4\%], respectively). It should, however, be noted that the lenalidomide starting dose in MDS-005 was adjusted based on each patient's creatinine clearance, which could have introduced a bias in the relationship between cumulative dose and response.

The most common reasons for lenalidomide dose adjustments were neutropenia and thrombocytopenia. This finding is consistent with data from a phase II trial evaluating lenalidomide with or without EPO in RBC-TD patients with lower-risk non-del(5q)

Figure 1 Ratio of RBC-TI $\geq 8$ Weeks Benefit-Risk (A) and Clinical Benefit-Risk (B) per Cycle in Patients Receiving LEN or PBO
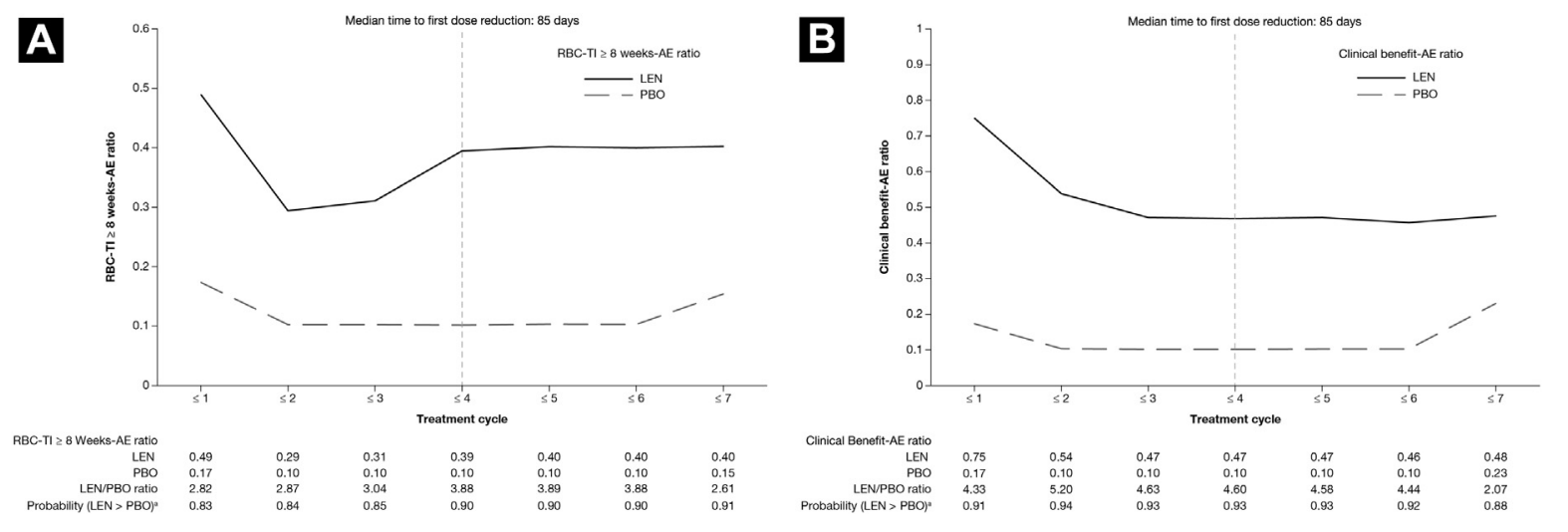

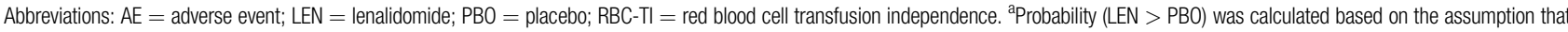
the benefit-risk ratio is higher for patients treated with LEN compared with PBO 


\section{Clinical Benefit-Risk Profile of Lenalidomide in Lower-Risk Non-del(5q) MDS}

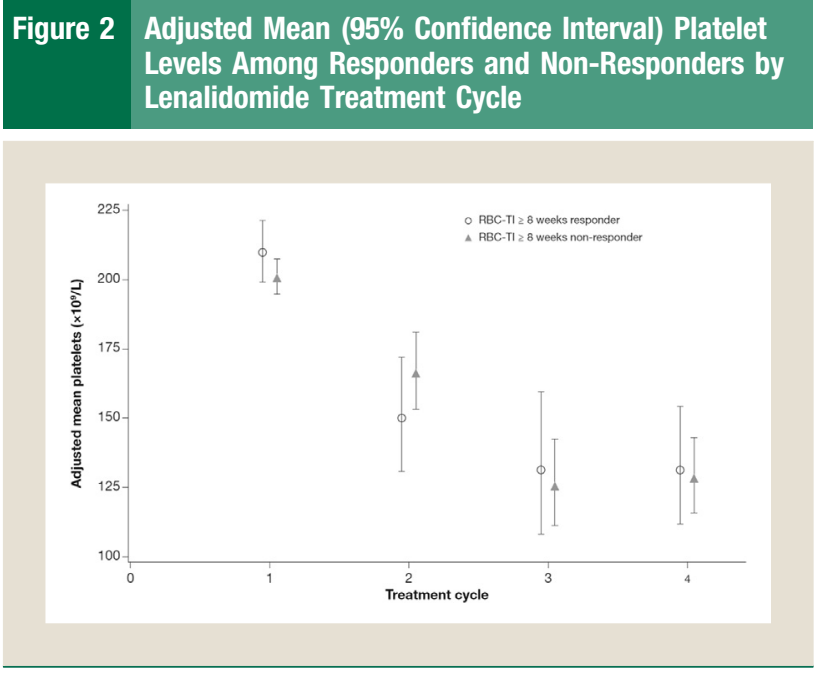

Abbreviation: RBC-TI = red blood cell transfusion independence.

MDS refractory to ESAs, in which cytopenias were the most common reason for dose reduction. ${ }^{20}$ Rates of discontinuation owing to neutropenia or thrombocytopenia were low in the MDS005 trial, suggesting that these events can be effectively managed with dose reduction or treatment interruption. In this population of lower-risk patients, cytopenias were more likely to develop as a TEAE than as a result of disease progression, allowing for management through dose modification.

The benefit-risk ratio in patients treated with lenalidomide was consistently higher than in patients receiving placebo. This suggests that, compared with placebo, the positive effects of lenalidomide treatment may outweigh the negative effects associated with an increased risk of hematologic TEAEs. In both treatment groups, the benefit-risk ratio remained below 1 due to a higher incidence of hematologic TEAEs reported, relative to the incidence of clinical benefit observed.

In our analysis, myelosuppression did not predict response to lenalidomide. Similar results were obtained in a retrospective analysis of data from 2 phase II studies of lenalidomide, in which a reduction in platelet or neutrophil count was associated with achievement of RBC-TI in patients with $\operatorname{del}(5 q)$, but not in those without del(5q). ${ }^{21}$ Thus, lenalidomide-related cytopenias may act as a surrogate marker of clonal suppression in patients with $\operatorname{del}(5 q)$ MDS, but cytopenias do not appear to predict response in patients with non-del $(5 q)$ MDS. It should be noted that patients were excluded from the MDS-005 trial if they had thrombocytopenia (ie, platelet count $<50 \times 10^{9} / \mathrm{L}$ ) or neutropenia (ie, absolute neutrophil count $\left.<0.5 \times 10^{9} / \mathrm{L}\right)$ at baseline. This may have influenced the lack of correlation between cytopenias and response. A preliminary analysis of response according to baseline platelet count showed that patients with low platelet counts $\left(50-100 \times 10^{9} / \mathrm{L}\right)$ were less likely to achieve RBC-TI $\geq 8$ weeks $(2 / 15[13.3 \%])$ than patients with platelet counts of $>100 \times 10 \%$ L (41/144 [28.5\%]).

This analysis is limited by its retrospective nature. Also, it should be noted that the lenalidomide starting dose was adjusted based on creatinine clearance, which could have influenced the relationship between cumulative dose and response. As patients with moderate renal impairment received a lower starting dose, the impact of the starting dose on other outcomes could not be assessed.

\section{Conclusion}

In summary, lenalidomide treatment was associated with a higher rate of clinical benefit than placebo in patients with non-del $(5 \mathrm{q})$ lower-risk MDS who were refractory to or ineligible for ESAs. Although treatment with lenalidomide was associated with an increased incidence of hematologic AEs, the overall benefit-risk ratio of lenalidomide treatment was more favorable than that of placebo. Managing hematologic AEs with appropriate dose reductions can help patients remain on treatment longer, thereby increasing the chance of achieving clinical benefit.

\section{Clinical Practice Points}

- Achievement of RBC-TI is a particularly meaningful endpoint for patients with RBC-TD anemia because prolonged RBC transfusion dependence is associated with significant clinical and HRQoL consequences.

- Our analysis supports the clinical benefit of lenalidomide in RBC-TD patients with non-del(5q) lower-risk MDS: a higher proportion of patients treated with lenalidomide achieved the composite endpoint of clinical benefit compared with placebo. This newly defined composite endpoint comprised the clinically meaningful endpoints of achievement of RBC-TI, reduced transfusion burden, increased $\mathrm{Hb}$, and $\mathrm{CyR}$. These data provide evidence that other measures of response in addition to RBC-TI may be valuable in the management of patients with non-del $(5 \mathrm{q})$ lower-risk MDS.

- Despite the occurrence of hematologic AEs, the benefit-risk profile consistently favored lenalidomide over placebo. This suggests that the positive effects of lenalidomide treatment may outweigh the negative effects associated with an increased risk of hematologic TEAEs.

- Patients undergoing lenalidomide dose reductions had a longer duration of treatment and were more likely to experience clinical benefit compared with patients without dose reductions. This finding suggests that lenalidomide dose reductions may allow patients who experience AEs to remain on treatment longer, leading to increased drug exposure and better outcomes.

- Overall, these data support the use of lenalidomide in patients with non-del(5q) lower-risk MDS who are ineligible for or refractory to ESAs.

\section{Acknowledgments}

This study was sponsored by Celgene Corporation, Summit, NJ. The authors received editorial and writing support, provided by Miriam de Boeck, from Excerpta Medica, supported by Celgene Corporation. The authors are fully responsible for all content and editorial decisions.

\section{Disclosure}

A.A. is a consultant for and has received honoraria from Celgene Corporation and Novartis; has received research funding from Celgene Corporation; and has presented at speakers' bureaus for Bristol-Myers Squibb, Celgene Corporation, Novartis, and Shire. 
N.G. is a consultant for and has received research funding and honoraria from Celgene Corporation and Novartis; and has received travel and accommodation expenses from Novartis. A.G. is a consultant for and has received honoraria from Celgene Corporation. S.L.G. is employed by and has equity ownership in COTA, Inc; has received research funding from Ariad Pharmaceuticals, Astellas Pharma, Bristol-Myers Squibb, Celator Pharmaceuticals, Celgene Corporation, Novartis, and Pfizer; is a consultant for and has presented at speakers' bureaus for Bristol-Myers Squibb and Novartis; has given expert testimony for Novartis; and has received honoraria, and travel and accommodation expenses from Pfizer. K.O. is a consultant for Celgene KK, JCR Pharma, and Sanofi; has received research funding from Takara Bio; has received honoraria from Alexion, Celgene KK, Kyowa Hakko Kirin, Sumitomo Dainippon Pharma, and Takeda; and has presented at speakers' bureaus for Celgene KK. J.W. is an employee of Celgene Corporation and has equity ownership in Celgene Corporation. V.S. has received research funding from Celgene Corporation and honoraria from Celgene Corporation, Janssen Pharmaceuticals, and Novartis. The remaining authors have stated that they have no conflicts of interest.

\section{Supplemental Data}

Supplemental materials, figures, and tables accompanying this article can be found in the online version at https://doi.org/10. 1016/j.clml.2018.12.012.

\section{References}

1. National Comprehensive Cancer Network. NCCN clinical practice guidelines in oncology: myelodysplastic syndromes (v2.2017), Available at: http://www.nccn. $\mathrm{org} / \mathrm{professionals/physician \_ gls/pdf/mds.pdf.} \mathrm{Accessed:} \mathrm{June} \mathrm{15,} 2017$.

2. Park S, Kelaidi C, Sapena R, et al. Early introduction of ESA in low risk MDS patients may delay the need for RBC transfusion: a retrospective analysis on 112 patients. Leuk Res 2010; 34:1430-6.

3. Platzbecker U, Symeonidis A, Oliva E, et al. ARCADE (20090160): a phase 3 randomized placebo-controlled double-blind trial of darbepoetin alfa in the treatment of anemia in patients with low or intermediate-1 risk myelodysplastic syndromes (MDS). Haematologica 2016; 101, abstract S128.
4. Fenaux P, Santini V, Aloe Spiriti MA, et al. Randomized, double-blind, placebo-controlled, multicentre study evaluating epoeitin alfa versus placebo in anemic patients with IPSS low- INT1 risk MDS. Haematologica 2016; 101, abstract P248.

5. Hellström-Lingberg E, Negrin R, Stein R, et al. Erythroid response to treatment with G-CSF plus erythropoietin for the anaemia of patients with myelodysplastic syndromes: proposal for a predictive model. Br J Haematol 1997; 99:344-51.

6. Santini V, Almeida A, Giagounidis A, et al. Randomized phase III study of lenalidomide versus placebo in RBC transfusion-dependent patients with lowerrisk non-del $(5 q)$ myelodysplastic syndromes and ineligible for or refractory to erythropoiesis-stimulating agents. J Clin Oncol 2016; 34:2988-96.

7. Malcovati L, Porta MG, Pascutto C, et al. Prognostic factors and life expectancy in myelodysplastic syndromes classified according to WHO criteria: a basis for clinical decision making. J Clin Oncol 2005; 23:7594-603.

8. Oliva EN, Finelli C, Santini V, et al. Quality of life and physicians' perception in myelodysplastic syndromes. Am I Blood Res 2012; 2:136-47.

9. List A, Kurtin S, Roe DJ, et al. Efficacy of lenalidomide in myelodysplastic syndromes. $N$ Engl J Med 2005; 352:549-57.

10. List A, Dewald G, Bennett J, et al. Lenalidomide in the myelodysplastic syndrome with chromosome 5q deletion. N Engl J Med 2006; 355:1456-65.

11. Fenaux P, Giagounidis A, Selleslag D, et al. A randomized phase 3 study of lenalidomide versus placebo in RBC transfusion-dependent patients with LowIntermediate-1-risk myelodysplastic syndromes. Blood 2011; 118:3765-6.

12. Almeida A, Fenaux P, Garcia-Manero G, et al. Safety profile of lenalidomide in patients with lower-risk myelodysplastic syndromes without $\operatorname{del}(5 \mathrm{q})$ : results of a phase 3 trial. Leuk Lymphoma 2018; 59:2135-43.

13. Cheson BD, Greenberg PL, Bennett JM, et al. Clinical application and proposal for modification of the International Working Group (IWG) response criteria in myelodysplasia. Blood 2006; 108:419-25.

14. Liang KY, Zeger SL. Longitudinal data analysis using generalized linear models. Biometrika 1986; 73:13-22.

15. Lefkopoulou M, Ryan 1. Global tests for multiple binary outcomes. Biometrics 1993; 49:975-88.

16. Oliva EN, Dimitrov BD, Benedetto F, D'Angelo A, Nobile F. Hemoglobin level threshold for cardiac remodelling and quality of life in myelodysplastic syndrome. Leuk Res 2005; 29:1217-9.

17. Szende A, Schaefer C, Goss TF, et al. Valuation of transfusion-free living in MDS: results of health utility interviews with patients. Health Qual Life Outcomes 2009; 7:81.

18. Sekeres MA, Swern AS, List AF, Fenaux P, Sugrue MM. Effect of lenalidomide (LEN) exposure on AML-free survival and overall survival in LEN-treated patients with IPSS low- or intermediate-1-risk myelodysplastic syndromes (MDS) with $\operatorname{del}(5 q)$. Blood 2015; 126, abstract 2870.

19. List AF, Giagounidis A, Backstrom JT, Fu T, Fenaux P. Early lenalidomide (LEN) dose intensity and durable RBC-transfusion independence (RBC-TI) in patients (pts) with low-/int-1-risk myelodysplastic syndromes (MDS) and del5q. J Clin Oncol 2011; 29, abstract 6522 .

20. Toma A, Kosmider O, Chevret S, et al. Lenalidomide with or without erythropoietin in transfusion-dependent erythropoiesis-stimulating agent-refractory lower risk MDS without 5q deletion. Leukemia 2016; 30:897-905.

21. Sekeres MA, Maciejewski JP, Giagounidis AA, et al. Relationship of treatmentrelated cytopenias and response to lenalidomide in patients with lower-risk myelodysplastic syndromes. J Clin Oncol 2008; 26:5943-9. 


\section{Clinical Benefit-Risk Profile of Lenalidomide in Lower-Risk Non-del(5q) MDS}

\section{Supplemental Data}

\section{Supplemental Methods \\ Modeling and Calculation of Benefit-Risk Ratios}

The bivariate generalized linear model has 2 assessment aspects to it, one which assesses benefit whereas the other assesses risk. Both benefit and risk can be modeled separately as described below:

(1) Let $Y_{i}^{(1)}$ be the response for benefit, which takes on the value 1 if the benefit criterion is met for a given subject and 0 otherwise.

Distribution : $Y_{i}^{(1)} \sim$ binomial $\left(n_{i}, \pi_{i}^{(1)}\right)$;

$$
\text { Link }: \log \left(\pi_{i}^{(1)}\right)=\eta_{i}^{(1)}
$$

Linear predictor : $\eta_{i}^{(1)}=\mu^{(1)}+\tau_{i}^{(1)}$
Furthermore, because we are also interested in comparing the treatment groups with respect to benefit-risk, an interaction term is also required, namely $\tau \theta_{i j}$. Assuming a binomial distribution and $\log \operatorname{link}$ as previously, this leads us to the following linear predictor:

$$
\text { Linear predictor : } \eta_{i j}=\mu+\tau_{i}+\theta_{j}+\tau \theta_{i j} \text {. }
$$

Note that by introducing the response type term, $\theta_{j}$, we can drop the superscripts (1) and (2) and replace it with subscript $\mathrm{j}$ to allow for the inclusion of response type.

Because we are modeling the benefit and risk simultaneously on the same set of subjects, recall that there is a shared $n_{i}$ for $Y_{i}^{(1)}$ and $Y_{i}^{(2)}$, so a correlation structure must be accounted for in estimating the terms in the model and their respective variances. Hence, we used generalized estimating equations with a compound symmetry structure as our working correlation in obtaining all our estimates. $^{1,2}$

To make a comparison of benefit-risk between the treatment groups, the following estimator can be constructed using the terms from the linear predictor:

$$
\widehat{T}=\left[\left(\widehat{\tau}_{i=1}+\widehat{\theta}_{j=1}+\widehat{\tau \theta}_{i=1, j=1}\right)-\left(\widehat{\tau}_{i=1}+\widehat{\theta}_{j=2}+\widehat{\tau \theta}_{i=1, j=2}\right)\right]-\left[\left(\widehat{\tau}_{i=2}+\widehat{\theta}_{j=1}+\widehat{\tau \theta}_{i=2, j=1}\right)-\left(\widehat{\tau}_{i=2}+\widehat{\theta}_{j=2}+\widehat{\tau \theta}_{i=2, j=2}\right)\right]
$$

where $\pi_{i}^{(1)}$ is the parameter for the proportion of benefit responders for the $i^{\text {th }}$ treatment group ( $\mathrm{i}=1$ for lenalidomide $[\mathrm{LEN}]$ and $\mathrm{i}=2$ for placebo [PBO]), $n_{i}$ is the sample size for the $i^{\text {th }}$ treatment group, and $\eta_{i}^{(1)}$ is the linear predictor for modeling the proportion of benefit responders on the log scale. The modeling terms we are interested in estimating involve the intercept, $\mu^{(1)}$, and the treatment effect, $\tau_{i}^{(1)}$.

2) Let $Y_{i}^{(2)}$ be the response for risk, which takes on the value 1 if a hematologic treatment-emergent adverse event (TEAE) occurs for a given subject and 0 otherwise.

$$
\text { Distribution : } Y_{i}^{(2)} \sim \operatorname{binomial}\left(n_{i}, \pi_{i}^{(2)}\right) \text {; }
$$

$$
\text { Link }: \log \left(\pi_{i}^{(2)}\right)=\eta_{i}^{(2)}
$$

$$
\text { Linear predictor : } \eta_{i}^{(2)}=\mu^{(2)}+\tau_{i}^{(2)}
$$

where $\pi_{i}^{(2)}$ is the parameter for the proportion of hematological TEAEs for the $i^{\text {th }}$ treatment group ( $i=1$ for LEN and $i=2$ for $\mathrm{PBO}$ ), and all other terms follow the same sort of interpretation as described for the previous model, but as applied to assessing the risk.

As we are interested in modeling benefit-risk simultaneously, we can introduce an additional modeling term that we will name $\theta_{j}$ ( $j=1$ for the benefit-response type, $j=2$ for the risk-response type). where $\widehat{T} \sim N\left(\mu_{T}, \sigma_{T}^{2}\right)$.

The terms contained within the first squared bracket, namely $\widehat{T}_{L E N}=\left[\left(\widehat{\tau}_{i=1}+\widehat{\theta}_{j=1}+\widehat{\tau \theta}_{i=1, j=1}\right)-\left(\widehat{\tau}_{i=1}+\widehat{\theta}_{j=2}+\right.\right.$ $\widehat{\tau \theta}_{i=1, j=2)}$ ], is the benefit-risk associated with the LEN group. When exponentiating $\widehat{T}_{L E N}$, the benefit-risk estimates will be on the ratio scale (ie, benefit:risk) as a log link function was used in the model.

The terms contained within the second squared bracket, namely $\widehat{T}_{P B O}=\left[\left(\widehat{\tau}_{i=2}+\widehat{\theta}_{j=1}+\widehat{\tau \theta}_{i=2, j=1}\right)-\left(\widehat{\tau}_{i=2}+\widehat{\theta}_{j=2}+\right.\right.$ $\widehat{\tau \theta}_{i=2, j=2)}$, is the benefit-risk associated with the PBO group. Likewise, when exponentiating $\widehat{T}_{P B O}$, the benefit-risk estimates will be on the ratio scale.

To obtain the relevant probabilities that the benefit-risk data from this study supports the hypothesis that the LEN group has a greater benefit-risk than the PBO group in terms of the unknown mean parameter $\mu_{T}$ [ie, $\operatorname{Pr}(\widehat{T}>0)$ ], we simply apply the model to the observed data to obtain the estimates for the mean $\left(\right.$ est $\left._{\mu_{T}}\right)$ and standard error $\left(\sqrt{\text { est }_{\sigma_{T}^{2}}}\right)$.

Once the above estimates are obtained the $\operatorname{Pr}(\widehat{T}>0)=$ $\Phi\left(\frac{\text { est }_{T_{T}}}{\sqrt{\text { est }_{\sigma_{T}^{2}}^{2}}}\right)$, where $\Phi(\bullet)$ is the cumulative normal density function, is calculated.

The model and the calculation of the probabilities described above were performed for each treatment cycle where cumulative events for both benefit and risk were incorporated in our assessments.

Note that using the maximum likelihood estimates of the benefit-risk ratios of LEN relative to $\mathrm{PBO}$ to construct a 
probability statement under an assumed asymptotic distribution is analogous to using a posterior distribution of a relative difference of the benefit-risk ratios between the 2 treatment groups with a non-informative prior.

Because the estimated within-subject correlation between the benefit endpoints and the risk endpoint was estimated to be very small, a beta-binomial model with a beta $(1 / 3,1 / 3)$ noninformative prior $^{3}$ was used as a sensitivity analysis assuming independence (ie, zero within subject correlation) between the benefit endpoints and the risk endpoint. The results from using the beta-binomial model to establish a formal posterior distribution were consistent with our bivariate modeling approach, although it yielded slightly more favorable probabilities for LEN than the bivariate modeling approach.

The generalized linear model procedure (PROC GENMOD) in SAS, Version 9.1 (SAS Institute Inc, Cary, NC) was used for these analyses.

\section{Analysis of Platelet Levels According to Response}

Adjusted mean platelet levels during cycles 1 to 4 were assessed according to response (red blood cell transfusion independence [RBC-TI] $\geq 8$ weeks) using a linear mixed model for repeated measures. Prior to modeling, a log transformation was applied to both post-treatment platelet responses and baseline platelet values. The linear mixed model contained a cell means parameterization for RBCTI (responder vs. non-responder) and cycle as fixed effects, and an unstructured within-subject covariance. Ninety-five percent confidence intervals $(95 \% \mathrm{CI})$ for the adjusted means were constructed as the least squares means \pm 2 standard errors. An additional exploratory analysis of patients with isolated anemia and non-isolated anemia was also conducted. For this analysis, the same approach was taken with respect to employing a linear mixed model for repeated measures, but with a cell means parameterization for RBC-TI, isolated anemia (yes vs. no), and cycle. The mixed procedure (PROC MIXED) in SAS, Version 9.1 was used for these analyses (SAS Institute Inc). 


\section{Clinical Benefit-Risk Profile of Lenalidomide in Lower-Risk Non-del(5q) MDS}

\section{Supplemental Figure 1 Timing of Clinical Benefit Response Measurements in Patients in the MDS-005 Study}

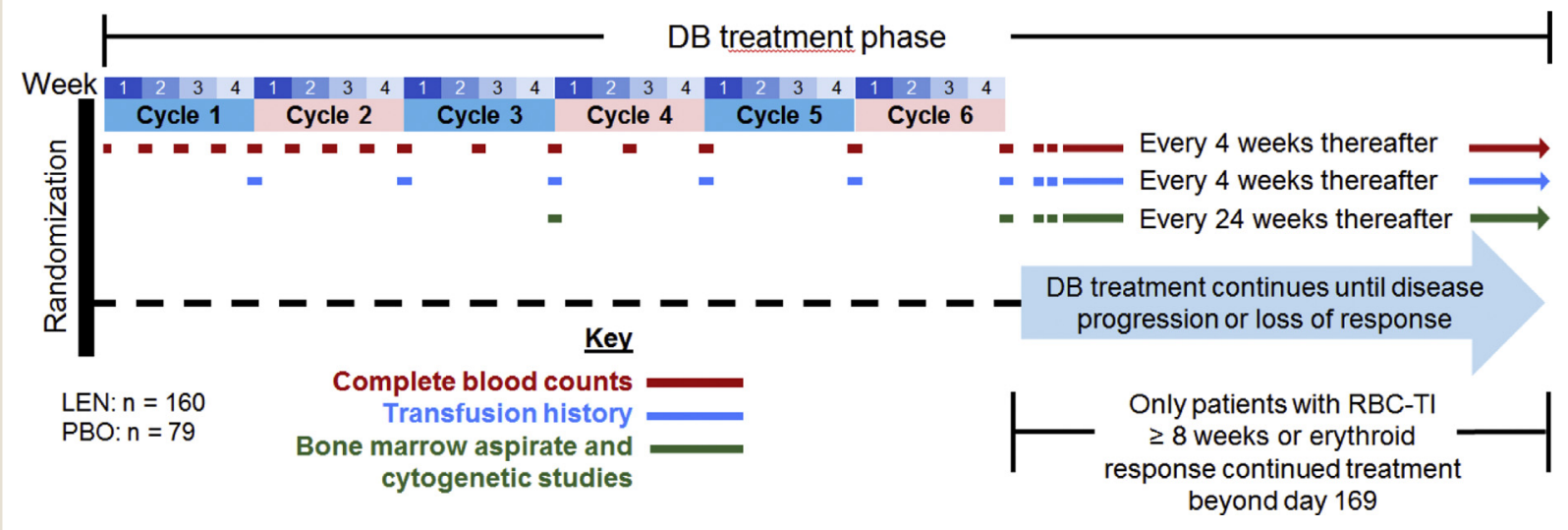

Abbreviations: $\mathrm{DB}=$ double-blinded; LEN = lenalidomide; $\mathrm{PBO}=$ placebo; RBC-TI = red blood cell transfusion independence.

\section{Supplemental Figure 2 Adjusted Mean (95\% Confidence Interval) Platelet Levels Among Responders and Non-Responders With} Isolated Anemia and Non-isolated Anemia by Lenalidomide Treatment Cycle

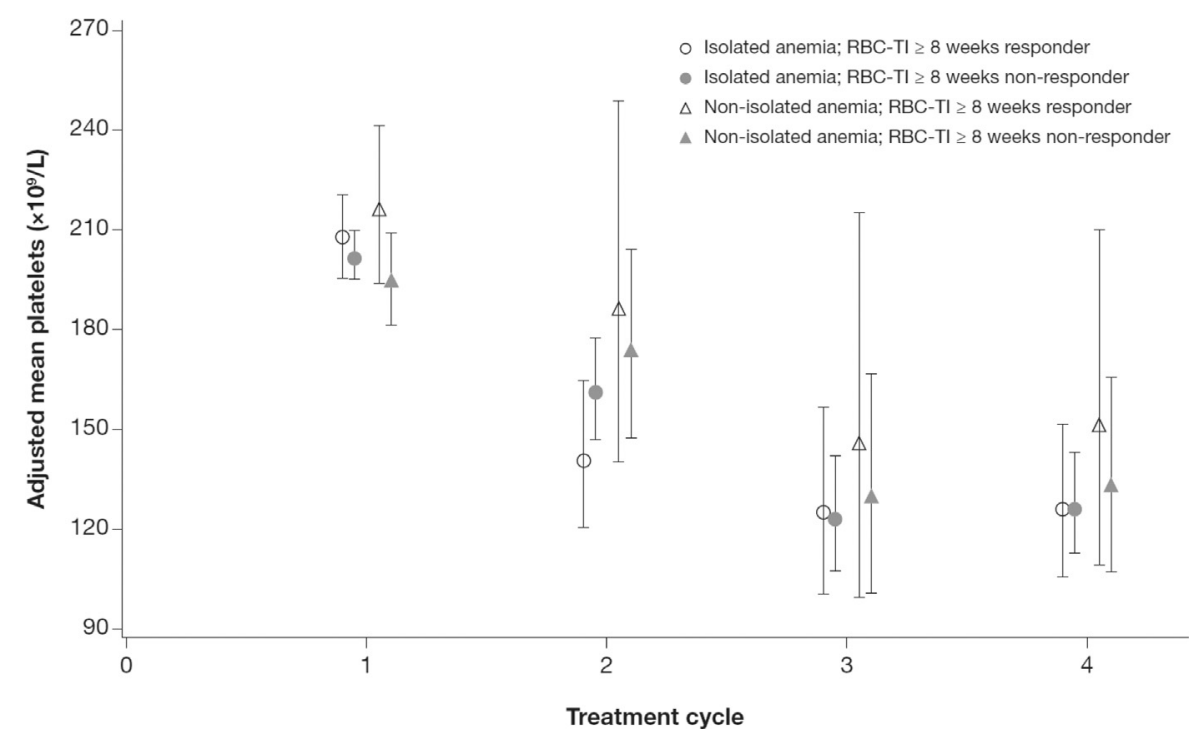

Abbreviation: RBC-TI = red blood cell transfusion independence. 


\section{Guillermo Garcia-Manero et al}

\begin{tabular}{|c|c|c|}
\hline Characteristic & $\geq 1$ Dose Reduction $(n=77$ ) & No Dose Reduction $(n=83)$ \\
\hline Median age, y (range) & $70(50-85)$ & $71(46-87)$ \\
\hline Male, $n(\%)$ & $52(67.5)$ & $56(67.5)$ \\
\hline Median time since diagnosis, y (range) & $3.3(0.2-29.6)$ & $2.5(0.1-23.9)$ \\
\hline \multicolumn{3}{|l|}{ IPSS risk, n (\%) } \\
\hline Low & $37(48.1)$ & $48(57.8)$ \\
\hline Int-1 & $40(51.9)$ & $35(42.2)$ \\
\hline Median RBC transfusion burden, units (range) & $3.0(1.8-8.8)$ & $3.0(1.8-6.8)$ \\
\hline \multicolumn{3}{|l|}{ Prior MDS therapy, n (\%) } \\
\hline No & $10(13.0)$ & $15(18.1)$ \\
\hline Yes & $67(87.0)$ & $68(81.9)$ \\
\hline \multicolumn{3}{|l|}{ Prior ESA therapy, n (\%) } \\
\hline No & $13(16.9)$ & $22(26.5)$ \\
\hline Yes & $64(83.1)$ & $61(73.5)$ \\
\hline Median bone marrow blast, \% (range) & $3.0(0-9.5)$ & $3.0(0-8.5)$ \\
\hline Median ANC, $\times 10^{9} / \mathrm{L}$ (range) & $2.4(0.5-12.2)$ & $2.4(0.5-7.7)$ \\
\hline Median platelet count, $\times 10^{9} / \mathrm{L}$ (range) & $224(43-588)$ & $263(51-746)$ \\
\hline Median Hb, g/dL (range) & $8.6(5.8-12.3)$ & $8.7(5.5-11.3)$ \\
\hline
\end{tabular}

Abbreviations: ANC = absolute neutrophil count; ESA = erythropoiesis-stimulating agent; Hb = hemoglobin; Int = Intermediate; IPSS = International Prognostic Scoring System; MDS = myelodysplastic syndrome; RBC $=$ red blood cell.

\section{References}

1. Lefkopoulou M, Ryan L. Global tests for multiple binary outcomes. Biometrics $1993 ; 49: 975-88$.
2. Liang KY, Zeger SL. Longitudinal data analysis using generalized linear models. Biometrika 1986; 73:13-22.

3. Kerman J. Neutral noninformative and informative conjugate beta and gamma prior distributions. Electron J Stat 2011; 5:1450-70. 